\title{
Exponentiated extended Weibull-power series class of distributions
}

\author{
S. Tahmasebi ${ }^{1}$ and A. A. Jafari*2 \\ ${ }^{1}$ Department of Statistics, Persian Gulf University, Bushehr, Iran \\ ${ }^{2}$ Department of Statistics, Yazd University, Yazd, Iran
}

\begin{abstract}
In this paper, we introduce a new class of distributions by compounding the exponentiated extended Weibull family and power series family. This distribution contains several lifetime models such as the complementary extended Weibull-power series, generalized exponential-power series, generalized linear failure rate-power series, exponentiated Weibull-power series, generalized modified Weibull-power series, generalized Gompertz-power series and exponentiated extended Weibull distributions as special cases. We obtain several properties of this new class of distributions such as Shannon entropy, mean residual life, hazard rate function, quantiles and moments. The maximum likelihood estimation procedure via a EM-algorithm is presented.
\end{abstract}

Keywords: EM-algorithm, Exponentiated family, Maximum likelihood estimation, Power series distributions.

\section{Introduction}

$\mathrm{T}$ He extended Weibull (EW) family contains various well-known distributions such as exponential, Pareto, Gompertz, Weibull, linear failure rate (Barlow, 1968), modified Weibull (Lai et al., 2003), additive Weibull (Xie and Lai, 1995; Almalki and Yuan, 2013) and Chen (Chen, 2000) distributions. For more details see Nadaraiah and Kotz (2005) and Pham and Lai (2007).

Using the given method by Gupta and Kundu (1999), the EW family can be generalized. We call it exponentiated extended Weibull (EEW) distribution. The cumulative distribution function (cdf) of this distribution is

$$
G(x ; \alpha, \beta, \boldsymbol{\Theta})=\left[1-e^{-\alpha H(x ; \boldsymbol{\Theta})}\right]^{\beta}, \alpha>0, \beta>0, x \geq 0,
$$

and its probability density function (pdf) is

$$
\begin{aligned}
\mathrm{g}(x ; \alpha, \beta, \boldsymbol{\Theta})= & \alpha \beta h(x ; \boldsymbol{\Theta}) e^{-\alpha H(x ; \boldsymbol{\Theta})} \\
& \times\left[1-e^{-\alpha H(x ; \boldsymbol{\Theta})}\right]^{\beta-1},
\end{aligned}
$$

\footnotetext{
*Corresponding author: aajafari@yazd.ac.ir

where $\boldsymbol{\Theta}$ is a vector of parameters, and $H(x ; \boldsymbol{\Theta})$ is a non-negative, continuous, monotone increasing, differentiable function of $x$ such that $H(x ; \boldsymbol{\Theta}) \rightarrow 0$ as $x \rightarrow 0^{+}$and $H(x ; \boldsymbol{\Theta}) \rightarrow \infty$ as $x \rightarrow \infty$. It is denoted by $\operatorname{EEW}(\alpha, \beta, \boldsymbol{\Theta})$.

The EEW distribution is a flexible family and contains many exponentiated distributions such as generalized exponential (Gupta and Kundu, 1999), exponentiated Weibull (Mudholkar and Srivastava, 1993), generalized Rayleigh (Surles and Padgett, 2001; Kundu and Raqab, 2005), generalized modified Weibull (Carrasco et al., 2008), generalized linear failure rate (Sarhan and Kundu, 2009), and generalized Gompertz (El-Gohary et al., 2013) distributions.

In recent years, many distributions to model lifetime data have been introduced. The basic idea of introducing these models is that a lifetime of a system with $N$ (discrete random variable) components and the positive continuous random variable, say $X_{i}$ (the lifetime of $i$ th component), can be denoted by the non-negative random variable $Y=\min \left(X_{1}, \ldots, X_{N}\right)$ or $Y=\max \left(X_{1}, \ldots, X_{N}\right)$, based on whether the components are series or parallel. 
In this paper, we compound the EEW family and power series distributions, and introduce a new class of distribution. This class of distributions can be applied to reliability problems and its some properties are investigated in this paper. We call it exponentiated extended Weibull- power series (EEWPS) class of distributions. In similar way, some distributions are proposed in literature: the exponential-power series (EP) distribution by Chahkandi and Ganiali (2009), Weibull-power series (WPS) distributions by Morais and Barreto-Souza (2011), generalized exponential-power series (GEP) distribution by Mahmoudi and Jafari (2012), complementary exponential power series by Flores et al. (2013), extended Weibull-power series (EWPS) distribution by Silva et al. (2013), double bounded Kumaraswamypower series by Bidram and Nekoukhou (2013), Burrpower series by Silva and Cordeiro (2013), generalized linear failure rate-power series (GLFRP) distribution by Alamatsaz and Shams (2014), Birnbaum-Saunderspower series distribution by Bourguignon et al. (2014), linear failure rate-power series by Mahmoudi and Jafari (2014), and complementary extended Weibull-power series by Cordeiro and Silva (2014). Similar procedures are used by Roman et al. (2012), Lu and Shi (2011), Nadarajah et al. (2015) and Louzada et al. (2014). For compounding continuous distributions with discrete distributions, Nadarajah et al. (2013) introduced the package Compounding in $\mathrm{R}$ software (R Development Core Team, 2014).

We provide three motivations for the EEWPS class of distributions, which can be applied in some interesting situations as follows: (i) this new class of distributions due to the stochastic representation $Y=\max \left(X_{1}, \ldots\right.$ ,$\left.X_{N}\right)$, can arises in parallel systems with identical components, where each component has the EEW distribution lifetime. This model appears in many industrial applications and biological organisms which the lifetime of the event is only the maximum ordered lifetime value among all causes. (ii) The EEWPS class of distributions gives a reasonable parametric fit to some modeling phenomenon with non-monotone hazard rates such as the bathtub-shaped, unimodal and increasing-decreasingincreasing hazard rates, which are common in reliability and biological studies. (iii) The time to the last failure can be appropriately modeled by the EEWPS class of distributions.

The remainder of this paper is organized as follows: the pdf and failure rate function of the new class of distributions are given in Section 2 The special cases of the EEWPS distribution are considered in Section 3 Some properties such as quantiles, moments, order statistics, Shannon entropy and mean residual life are given in Section 4 Estimation of parameters by maximum likelihood are discussed in Section 5. Application to a real data set is presented in Section 6

\section{Introducing new family}

A discrete random variable, $N$ is a member of power series distributions (truncated at zero) if its probability mass function (pmf) is given by

$$
p_{n}=P(N=n)=\frac{a_{n} \lambda^{n}}{C(\lambda)}, n=1,2, \ldots,
$$

where $a_{n} \geq 0, C(\lambda)=\sum_{n=1}^{\infty} a_{n} \lambda^{n}$, and $\lambda \in(0, s)$ is chosen in a way such that $C(\lambda)$ is finite and its first, second and third derivatives are defined and shown by $C^{\prime}($.$) ,$ $C^{\prime \prime}($.$) and C^{\prime \prime \prime}($.$) , respectively. The term "power se-$ ries distribution" is generally credited to Noack (1950). This family of distributions includes many of the most common distributions, including the binomial, Poisson, geometric, negative binomial, logarithmic distributions. For more details about power series distributions, see Iohnson et al. (2005), page 75.

Theorem 2.1. Let $N$ be a random variable denoting the number of failure causes which it is a member of power series distributions with pmf in (3). Also, For given $N$, let $X_{1}, X_{2}, \ldots, X_{N}$ be independent identically distributed random variables from $E E W$ distribution with $p d f$ in (2). Then $X_{(N)}=\max _{1 \leq i \leq N}\left\{X_{i}\right\}$ has EEWPS class of distributions is denoted by $\operatorname{EEWPS}(\alpha, \beta, \lambda, \boldsymbol{\Theta})$ and has the following $p d f$ :

$$
\begin{aligned}
f(x)= & \alpha \beta \lambda h(x ; \boldsymbol{\Theta}) e^{-\alpha H(x ; \boldsymbol{\Theta})}\left(1-e^{-\alpha H(x ; \boldsymbol{\Theta})}\right)^{\beta-1} \\
& \times \frac{C^{\prime}\left(\lambda\left(1-e^{-\alpha H(x ; \boldsymbol{\Theta})}\right)^{\beta}\right)}{C(\lambda)}, x>0 .
\end{aligned}
$$

Proof. The conditional cdf of $X_{(N)} \mid N=n$ has $\operatorname{EEW}(\alpha, n \beta, \boldsymbol{\Theta})$. Hence,

$$
P\left(X_{(N)} \leq x, N=n\right)=\frac{a_{n} \lambda^{n}}{C(\lambda)}\left[1-e^{-\alpha H(x ; \boldsymbol{\Theta})}\right]^{n \beta},
$$

and the marginal cdf of $X_{(N)}$ is

$$
F(x)=\frac{C\left(\lambda\left(1-e^{-\alpha H(x ; \boldsymbol{\Theta})}\right)^{\beta}\right)}{C(\lambda)}, x>0 .
$$

The derivative of $F$ with respect to $x$ is (4). Therefore, $X_{(N)}$ has EEWPS distribution.

Proposition 1. The pdf of EEWPS class can be expressed as infinite linear combination of density of order distribution, i.e. it can be written as

$$
f(x)=\sum_{n=1}^{\infty} p_{n} g_{(n)}(x ; \alpha, n \beta, \boldsymbol{\Theta}),
$$

where $g_{(n)}(x ; \alpha, n \beta, \boldsymbol{\Theta})$ is the pdf of EEW distribution with parameters $\alpha, n \beta$ and $\boldsymbol{\Theta}$. 
Proof. Consider $t=1-e^{-\alpha H(x ; \boldsymbol{\Theta})}$. So

$$
\begin{aligned}
f(x) & =\alpha \beta \lambda h(x ; \boldsymbol{\Theta}) e^{-\alpha H(x ; \boldsymbol{\Theta})} t^{\beta-1} \frac{C^{\prime}\left(\lambda t^{\beta}\right)}{C(\lambda)} \\
& =\alpha \beta \lambda h(x ; \boldsymbol{\Theta}) e^{-\alpha H(x ; \boldsymbol{\Theta})} t^{\beta-1} \frac{\sum_{n=1}^{\infty} n a_{n}\left(\lambda t^{\beta}\right)^{n-1}}{C(\lambda)} \\
& =\sum_{n=1}^{\infty} \frac{a_{n} \lambda^{n}}{C(\lambda)} n \alpha \beta h(x ; \boldsymbol{\Theta}) e^{-\alpha H(x ; \boldsymbol{\Theta})} t^{n \beta-1} \\
& =\sum_{n=1}^{\infty} p_{n} g_{(n)}(x ; \alpha, n \beta, \boldsymbol{\Theta}) .
\end{aligned}
$$

Proposition 2. The limiting distribution of $\operatorname{EEWPS}(\beta, \lambda, \Theta)$ when $\lambda \rightarrow 0^{+}$is

$$
\lim _{\lambda \rightarrow 0^{+}} F(x)=\left[1-e^{-\alpha H(x ; \Theta)}\right]^{c \beta},
$$

which is a EEW distribution with parameters $\alpha, c \beta$ and $\boldsymbol{\Theta}$, where $c=\min \left\{n \in \mathbb{N}: a_{n}>0\right\}$.

Proof. Consider $t=1-e^{-\alpha H(x ; \boldsymbol{\Theta})}$. So

$$
\begin{aligned}
\lim _{\lambda \rightarrow 0^{+}} F(x) & =\lim _{\lambda \rightarrow 0^{+}} \frac{C\left(\lambda t^{\beta}\right)}{C(\lambda)}=\lim _{\lambda \rightarrow 0^{+}} \frac{\sum_{n=1}^{\infty} a_{n} \lambda^{n} t^{n \beta}}{\sum_{n=1}^{\infty} a_{n} \lambda^{n}} \\
& =\lim _{\lambda \rightarrow 0^{+}} \frac{a_{c} t^{c \beta}+\sum_{n=c+1}^{\infty} a_{n} \lambda^{n-c} t^{n \beta}}{a_{c}+\sum_{n=c+1}^{\infty} a_{n} \lambda^{n-c}}=t^{c \beta} .
\end{aligned}
$$

Proposition 3. The hazard rate function of the EEWPS class of distributions is given by

$$
r(x)=\frac{\alpha \lambda \beta h(x ; \Theta)(1-t) t^{\beta-1} C^{\prime}\left(\lambda t^{\beta}\right)}{C(\lambda)-C\left(\lambda t^{\beta}\right)}
$$

where $t=1-e^{-\alpha H(x ; \boldsymbol{\Theta})}$.

Proof. Using (4), (6) and definition of hazard rate function as $r(x)=f(x) /(1-F(x)$, the proof is obvious.

\section{Special cases}

In this Section, we consider some special cases of the EEWPS distribution.

\subsection{Complementary extended Weibull power series}

If $\beta=1$, then the pdf in (4) becomes to

$$
f(x)=\alpha \lambda h(x ; \boldsymbol{\Theta}) e^{-\alpha H(x ; \boldsymbol{\Theta})}
$$

$$
\times \frac{C^{\prime}\left(\lambda\left(1-e^{-\alpha H(x ; \boldsymbol{\Theta})}\right)\right)}{C(\lambda)}, x>0,
$$

which is the pdf of complementary extended Weibull power series (CEWPS) class of distributions introduced by Cordeiro and Silva (2014).

\subsection{Generalized exponential-power series}

If $H(x ; \boldsymbol{\Theta})=x$, then the pdf in (4) becomes to

$f(x)=\alpha \beta \lambda e^{-\alpha x}\left(1-e^{-\alpha x}\right)^{\beta-1} \frac{C^{\prime}\left(\lambda\left(1-e^{-\alpha x}\right)^{\beta}\right)}{C(\lambda)}, x>0$.

which is the pdf of generalized exponential-power series (GEPS) class of distributions introduced by Mahmoudi and Jafari (2012). The GEPS class of distributions contains complementary exponentiated exponential-geometric distribution introduced by Louzada et al. (2013), complementary exponentialgeometric distribution introduced by Louzada et al. (2011), Poisson-exponential distribution introduced by Cancho et al. (2011) and Louzada-Neto et al. (2011), complementary exponential-power series class of distributions introduced by Flores et al. (2013), generalized exponential distribution introduced by Gupta and Kundu (1999) and generalized exponentialgeometric distribution introduced by Bidram et al. (2013) .

\subsection{Generalized linear failure rate-power se- ries}

If $H(x ; \Theta)=\frac{a x}{\alpha}+\frac{b x^{2}}{2 \alpha}$, then the pdf in (4) becomes to

$$
\begin{aligned}
f(x)= & \beta \lambda(a+b x) e^{-a x-\frac{b x^{2}}{2}}\left(1-e^{-a x-\frac{b x^{2}}{2}}\right)^{\beta-1} \\
& \times \frac{C^{\prime}\left(\lambda\left(1-e^{-a x-\frac{b x^{2}}{2}}\right)^{\beta}\right)}{C(\lambda)}, x>0 .
\end{aligned}
$$

which is the pdf of generalized linear failure ratepower series (GLFRPS) class of distributions introduced by Alamatsaz and Shams (2014). It is a modification of generalized linear failure rate distribution introduced by Sarhan and Kundu (2009) and generalized linear failure rate- geometric distribution introduced by Nadarajah et al. (2014). If $b=0$, it becomes to GEPS class of distributions. Also, If $\beta=1$, it becomes to linear failure rate-power series introduced by Mahmoudi and Jafari (2014).

\subsection{Exponentiated Weibull- power series}

If $H(x ; \Theta)=x^{\gamma}$, then the pdf in (4) becomes to

$$
f(x)=\alpha \beta \lambda \gamma x^{\gamma-1} e^{-\alpha x^{\gamma}}\left(1-e^{-\alpha x^{\gamma}}\right)^{\beta-1}
$$




$$
\times \frac{C^{\prime}\left(\lambda\left(1-e^{-\alpha x^{\gamma}}\right)^{\beta}\right)}{C(\lambda)}, x>0 .
$$

which is the pdf of exponentiated Weibull-power series (EWPS) class of distributions introduced by Mahmoudi and Shiran (2012). It is a modification of exponentiated Weibull distribution introduced by Mudholkar and Srivastava (1993). It is contain the complementary Weibull geometric distribution introduced by Tojeiro et al. (2014). Also, the MarshallOlkin extended Weibull distribution introduced by Cordeiro and Lemonte (2013) is a special case of EWPS.

\subsection{Generalized modified Weibull-power series}

If $H(x ; \boldsymbol{\Theta})=x^{\gamma} \exp (\tau x)$, then the pdf in (4) becomes to

$$
\begin{aligned}
f(x)= & \alpha \beta \lambda x^{\gamma-1}(\gamma+\tau x) e^{\tau x-\alpha x^{\gamma} \exp (\tau x)} \\
& \times \frac{C^{\prime}\left(\lambda\left(1-e^{-\alpha x^{\gamma} \exp (\tau x)}\right)^{\beta}\right)}{\left(1-e^{-\alpha x^{\gamma} \exp (\tau x)}\right)^{1-\beta} C(\lambda)}, \quad x>0,
\end{aligned}
$$

and we call generalized modified Weibull-power series (GMWPS) class of distributions. It is contained the generalized modified Weibull distribution introduced by Carrasco et al. (2008). If $\tau=0$, then GMWPS class of distributions becomes to EWPS class of distributions.

\subsection{Generalized Gompertz- power series}

If $H(x ; \Theta)=\frac{1}{\gamma}\left(e^{\gamma x}-1\right)$, then the pdf in (4) becomes to

$$
\begin{aligned}
f(x)= & \alpha \beta \lambda e^{\gamma x} e^{-\frac{\alpha}{\gamma}\left(e^{\gamma x}-1\right)}\left(1-e^{-\frac{\alpha}{\gamma}\left(e^{\gamma x}-1\right)}\right)^{\beta-1} \\
& \times \frac{C^{\prime}\left(\lambda\left(1-e^{-\frac{\alpha}{\gamma}\left(e^{\gamma x}-1\right)}\right)^{\beta}\right)}{C(\lambda)}, x>0 .
\end{aligned}
$$

and we call generalized Gompertz-power series class of distributions. It is contained the generalized Gompertz distribution introduced by El-Gohary et al. (2013).

\section{Statistical properties}

In this section, some properties of EEWPS class of distributions such as quantiles, moments, order statistics, Shannon entropy and mean residual life are derived. Using (7), we can obtain

$$
F(x)=\sum_{n=1}^{\infty} p_{n} G_{(n)}(x ; \alpha, n \beta, \boldsymbol{\Theta})=\sum_{n=1}^{\infty} p_{n} t^{n \beta},
$$

where $t=1-e^{-\alpha H(x ; \boldsymbol{\Theta})}$. Based on the mathematical quantities of the baseline pdf $g_{(n)}(x ; \alpha, n \beta, \boldsymbol{\Theta})$, we can obtain some statistical quantities such as ordinary and incomplete moments, generating function and mean deviations of this family of distributions.

\subsection{Quantiles and Moments}

Let

$$
X=G^{-1}\left(\frac{C^{-1}(C(\lambda) U)}{\lambda}\right),
$$

where $U$ has a uniform distribution on $(0,1), G^{-1}(y)=$ $H^{-1}\left[-\frac{1}{\alpha} \ln \left(1-y^{\frac{1}{\beta}}\right)\right]$ and $C^{-1}($.$) is the inverse function$ of $C($.$) . Then X$ has the EEWPS $(\alpha, \beta, \lambda, \Theta)$ distribution. This result helps in simulating data from the EEWPS distribution with generating uniform distribution data.

Theorem 4.1. Consider $X \sim \operatorname{EEWPS}(\alpha, \beta, \lambda, \boldsymbol{\Theta})$. Then the moment generating function of EEWPS is

$$
M_{X}(t)=\sum_{n=1}^{\infty} \sum_{j=0}^{\infty} p_{n}\left(\begin{array}{c}
n \beta \\
j+1
\end{array}\right)(-1)^{j} M_{Y}(t),
$$

where $Y$ has $\operatorname{EEW}(\alpha(j+1), 1, \boldsymbol{\Theta})$.

Proof. The Laplace transform of the EEWPS class can be expressed as

$$
L(s)=E\left(e^{-s X}\right)=\sum_{n=1}^{\infty} P(N=n) L_{n}(s),
$$

where $L_{n}(s)$ is the Laplace transform of EEW distribution with parameters $\alpha, n \beta$ and $\Theta$ given as

$$
\begin{aligned}
L_{n}(s)= & \int_{0}^{+\infty} e^{-s x} n \alpha \beta h(x ; \boldsymbol{\Theta}) e^{-\alpha H(x ; \boldsymbol{\Theta})} \\
& \times\left[1-e^{-\alpha H(x ; \boldsymbol{\Theta})}\right]^{n \beta-1} d x \\
= & n \alpha \beta \int_{0}^{+\infty} e^{-s x} h(x ; \boldsymbol{\Theta}) \\
& \times \sum_{j=0}^{\infty}\left(\begin{array}{c}
n \beta-1 \\
j
\end{array}\right)(-1)^{j} e^{-(j+1) \alpha H(x ; \boldsymbol{\Theta})} d x \\
= & \sum_{j=0}^{\infty} n \beta\left(\begin{array}{c}
n \beta-1 \\
j
\end{array}\right)(-1)^{j} \int_{0}^{+\infty} \frac{\alpha(j+1)}{j+1} \\
& \times h(y ; \boldsymbol{\Theta}) e^{-(j+1) \alpha H(y ; \boldsymbol{\Theta})-s y d y} \\
= & \sum_{j=0}^{\infty}\left(\begin{array}{c}
n \beta \\
j+1
\end{array}\right)(-1)^{j} L_{1}(s),
\end{aligned}
$$

where $L_{1}(s)$ is the Laplace transform of the $\operatorname{EEW}(\alpha(j+$ $1), 1, \Theta)$. Therefore, the moment generating function of EEWPS is

$$
\begin{aligned}
M_{X}(t) & =\sum_{n=1}^{\infty} p_{n} L_{n}(-t) \\
& =\sum_{n=1}^{\infty} \sum_{j=0}^{\infty} p_{n}\left(\begin{array}{c}
n \beta \\
j+1
\end{array}\right)(-1)^{j} L_{1}(-t) \\
& =\sum_{n=1}^{\infty} \sum_{j=0}^{\infty} p_{n}\left(\begin{array}{c}
n \beta \\
j+1
\end{array}\right)(-1)^{j} M_{Y}(t) .
\end{aligned}
$$


Table 1: The four moments of EEWPS model.

\begin{tabular}{cccc|cccc}
\hline$\alpha$ & $\beta$ & $\lambda$ & $\gamma$ & $\mu_{1}$ & $\mu_{2}$ & $\mu_{3}$ & $\mu_{4}$ \\
\hline 0.3 & 0.3 & 0.2 & 2.0 & 0.936 & 1.594 & 3.520 & 9.164 \\
0.3 & 0.3 & 0.2 & 5.0 & 0.856 & 0.884 & 1.011 & 1.237 \\
0.3 & 0.3 & 0.8 & 2.0 & 1.656 & 3.719 & 9.722 & 28.292 \\
0.3 & 0.3 & 0.8 & 5.0 & 1.150 & 1.446 & 1.916 & 2.631 \\
0.3 & 2.0 & 0.2 & 2.0 & 2.192 & 5.446 & 14.976 & 44.841 \\
0.3 & 2.0 & 0.2 & 5.0 & 1.345 & 1.853 & 2.606 & 3.734 \\
0.3 & 2.0 & 0.8 & 2.0 & 2.835 & 8.733 & 28.694 & 99.531 \\
0.3 & 2.0 & 0.8 & 5.0 & 1.500 & 2.285 & 3.530 & 5.521 \\
0.8 & 0.3 & 0.2 & 2.0 & 0.573 & 0.598 & 0.808 & 1.289 \\
0.8 & 0.3 & 0.2 & 5.0 & 0.704 & 0.597 & 0.561 & 0.565 \\
0.8 & 0.3 & 0.8 & 2.0 & 1.014 & 1.394 & 2.232 & 3.979 \\
0.8 & 0.3 & 0.8 & 5.0 & 0.945 & 0.977 & 1.064 & 1.201 \\
0.8 & 2.0 & 0.2 & 2.0 & 1.342 & 2.042 & 3.439 & 6.306 \\
0.8 & 2.0 & 0.2 & 5.0 & 1.106 & 1.252 & 1.446 & 1.704 \\
0.8 & 2.0 & 0.8 & 2.0 & 1.736 & 3.275 & 6.589 & 13.997 \\
0.8 & 2.0 & 0.8 & 5.0 & 1.233 & 1.543 & 1.960 & 2.519 \\
2.0 & 0.3 & 0.2 & 2.0 & 0.362 & 0.239 & 0.204 & 0.206 \\
2.0 & 0.3 & 0.2 & 5.0 & 0.586 & 0.414 & 0.324 & 0.271 \\
2.0 & 0.3 & 0.8 & 2.0 & 0.641 & 0.558 & 0.565 & 0.637 \\
2.0 & 0.3 & 0.8 & 5.0 & 0.787 & 0.677 & 0.614 & 0.577 \\
2.0 & 2.0 & 0.2 & 2.0 & 0.849 & 0.817 & 0.870 & 1.009 \\
2.0 & 2.0 & 0.2 & 5.0 & 0.921 & 0.867 & 0.835 & 0.819 \\
2.0 & 2.0 & 0.8 & 2.0 & 1.098 & 1.310 & 1.667 & 2.239 \\
2.0 & 2.0 & 0.8 & 5.0 & 1.026 & 1.070 & 1.131 & 1.210 \\
\hline & & & & & & &
\end{tabular}

Theorem 4.2. The noncentral moment functions of EEWPS is

$$
\begin{aligned}
\mu_{r} & =\sum_{n=1}^{\infty} \frac{a_{n} \lambda^{n}}{C(\lambda)} \sum_{j=0}^{\infty}\left(\begin{array}{c}
n \beta \\
j+1
\end{array}\right)(-1)^{j} \mu_{r}^{\prime} \\
& =\sum_{n=1}^{\infty} \sum_{j=0}^{\infty} p_{n}\left(\begin{array}{c}
n \beta \\
j+1
\end{array}\right)(-1)^{j} \mu_{r}^{\prime},
\end{aligned}
$$

where $\mu_{r}^{\prime}=E\left[Y^{r}\right]$ and $Y$ has $\operatorname{EEW}(\alpha(j+1), 1, \boldsymbol{\Theta})$.

Proof. We can use $M_{X}(t)$ to obtain $\mu_{r}$. But from the direct calculation, proof is obvious.

Considering $H(x)=x^{\gamma}$ and $C(\lambda)=\lambda(1-\lambda)^{-1}$, we calculated the first four moments with different values of parameters for the EEWPS distribution using (17). Also, we computed these values from the direct definition by numerical integration. We found that the results are same. The values are given in Table 1.

\subsection{Order statistic}

Let $X_{1}, X_{2}, \ldots, X_{m}$ be a random sample of size $m$ from $\operatorname{EEWPS}(\alpha, \beta, \lambda, \Theta)$, then the pdf of the $i$ th order statistic, say $X_{i: m}$, is given by

$$
\begin{aligned}
f_{i: m}(x)= & \frac{m !}{(i-1) !(m-i) !} f(x)\left[\frac{C\left(\lambda t^{\beta}\right)}{C(\lambda)}\right]^{i-1} \\
& \times\left[1-\frac{C\left(\lambda t^{\beta}\right)}{C(\lambda)}\right]^{m-i}
\end{aligned}
$$

$$
\begin{aligned}
= & \frac{m !}{(i-1) !(m-i) !} f(x) \sum_{j=0}^{m-i}\left(\begin{array}{c}
m-i \\
j
\end{array}\right) \\
& \times(-1)^{j}\left[\frac{C\left(\lambda t^{\beta}\right)}{C(\lambda)}\right]^{j+i-1} \\
= & \frac{m !}{(i-1) !(m-i) !} \sum_{n=1}^{\infty} \sum_{j=0}^{m-i} p_{n} g_{(n)}(x ; \alpha, n \beta, \boldsymbol{\Theta}) \\
& \times\left(\begin{array}{c}
m-i \\
j
\end{array}\right)(-1)^{j}\left[\frac{C\left(\lambda t^{\beta}\right)}{C(\lambda)}\right]^{j+i-1} \\
= & \frac{m !}{(i-1) !(m-i) !} \sum_{n=1}^{\infty} \sum_{j=0}^{m-i} w_{j} p_{n} \\
& \times g_{(n)}(x ; \alpha, n \beta, \boldsymbol{\Theta})\left[\frac{C\left(\lambda t^{\beta}\right)}{C(\lambda)}\right]^{j+i-1},
\end{aligned}
$$

where $f$ is the pdf of EEWP class of distributions, $t=$ $1-e^{-\alpha H(x ; \boldsymbol{\Theta})}$ and $w_{j}=\left(\begin{array}{c}m-i \\ j\end{array}\right)(-1)^{j}$. Also, the cdf of $X_{i: m}$ is given by

$$
F_{i: m}(x)=\sum_{k=i}^{m} \sum_{j=0}^{m-k}(-1)^{j}\left(\begin{array}{c}
m-k \\
j
\end{array}\right)\left(\begin{array}{c}
m \\
k
\end{array}\right)\left[\frac{C\left(\lambda t^{\beta}\right)}{C(\lambda)}\right]^{j+k} .
$$

An analytical expression for $r$ th moment of order statistics $X_{i: m}$ is obtained as

$$
\begin{aligned}
E\left[X_{i: m}^{r}\right]= & \frac{m !}{(i-1) !(m-i) !} \sum_{n=1}^{\infty} \sum_{j=0}^{m-i} w_{j} p_{n} \\
& \times E\left[Z^{r}(F(Z))^{j+i-1}\right],
\end{aligned}
$$

where $Z$ has a EEW distribution with parameters $\alpha, n \beta$ and $\Theta$.

\subsection{Shannon entropy and mean residual life}

The maximum entropy method is a powerful technique in the field of probability and statistics. It is introduced by Iaynes (1957) and closely related to the Shannon's entropy. Also, it is applied in a wide variety of fields and used for the characterization of pdf's; see, for example, Kapur (1994), Soofi (2000) and Zografos and Balakrishnan (2009). Shore and Johnson (1980) treated the maximum entropy method axiomatically.

$$
\text { Consider a class of pdf's }
$$

$$
F=\left\{f(x ; \alpha, \beta, \lambda, \boldsymbol{\Theta}): E_{f}\left(T_{i}(X)\right)=\beta_{i}, i=0,1, \ldots ., m\right\}
$$

where $T_{1}(X), \ldots, T_{m}(X)$ are absolutely integrable functions with respect to $f$, and $T_{0}(X)=1$. Also, consider the shannon's entropy of none-negative continuous random variable $X$ with pdf $f$ defined by Shannon (1948) 
as

$$
H_{s h}(f)=E[-\log f(X)]=-\int_{0}^{+\infty} f(x) \log (f(x)) d x .
$$

The maximum entropy distribution is the pdf of the class $F$, denoted by $f^{M E}$ determined as

$$
f^{M E}(x ; \lambda, \beta, \boldsymbol{\Theta})=\arg \max _{f \in F} H_{s h}(f) .
$$

Now, suitable constraints are derived in order to provide a maximum entropy characterization for the class (18) based on Iaynes (1957). For this purpose, the next result plays an important role.

Proposition 4. Let $X$ has $\operatorname{EEWPS}(\alpha, \beta, \lambda, \Theta)$ with the $p d f$ given by (4). Then, $i$.

$$
\begin{aligned}
E & {\left[\log \left(C^{\prime}\left(\lambda\left(1-e^{-\alpha H(X ; \boldsymbol{\Theta})}\right)^{\beta}\right)\right)\right] } \\
= & \frac{\lambda}{C(\lambda)} E\left[C^{\prime}\left(\lambda\left(1-e^{-\alpha H(Y ; \boldsymbol{\Theta})}\right)^{\beta}\right)\right. \\
& \left.\times \log \left(C^{\prime}\left(\lambda\left(1-e^{-\alpha H(Y ; \boldsymbol{\Theta})}\right)^{\beta}\right)\right)\right],
\end{aligned}
$$

ii.

$$
\begin{array}{r}
E[\log (h(X ; \boldsymbol{\Theta}))]=\frac{\lambda}{C(\lambda)} E\left[C^{\prime}\left(\lambda\left(1-e^{-H(Y ; \boldsymbol{\Theta})}\right)^{\beta}\right)\right. \\
\times \log (h(Y ; \boldsymbol{\Theta}))],
\end{array}
$$

iii.

$$
\begin{aligned}
& E\left[\log \left(1-e^{-\alpha H(X ; \boldsymbol{\Theta})}\right)\right]=\frac{\lambda}{C(\lambda)} \\
& \quad \times E\left[C^{\prime}\left(\lambda\left(1-e^{-\alpha H(Y ; \boldsymbol{\Theta})}\right)^{\beta}\right) \log \left(1-e^{-\alpha H(Y ; \boldsymbol{\Theta})}\right)\right],
\end{aligned}
$$

where $Y$ follows the EEW distribution with the pdf in (2).

An explicit expression of Shannon entropy for EEWPS distribution is obtained as

$$
\begin{aligned}
H_{s h}(f) & =-\log (\alpha \beta \lambda)-\frac{\lambda}{C(\lambda)} E\left[C^{\prime}\left(\lambda\left(1-e^{-H(Y ; \boldsymbol{\Theta})}\right)^{\beta}\right)\right. \\
& \left.\times \log \left(C^{\prime}\left(\lambda\left(1-e^{-H(Y ; \boldsymbol{\Theta})}\right)^{\beta}\right)\right)\right] \\
& +\log [C(\lambda)]-(\beta-1) \frac{\lambda}{C(\lambda)} \\
& \times E\left[C^{\prime}\left(\lambda\left(1-e^{-H(Y ; \boldsymbol{\Theta})}\right)^{\beta}\right) \log \left(1-e^{-H(Y ; \boldsymbol{\Theta})}\right)\right] \\
& -\frac{\lambda}{C(\lambda)} E\left[C^{\prime}\left(\lambda\left(1-e^{-H(Y ; \boldsymbol{\Theta})}\right)^{\beta}\right) \log (h(Y ; \boldsymbol{\Theta}))\right] .
\end{aligned}
$$

Also, the mean residual life function of $X$ is given by

$$
\begin{aligned}
m(t) & =E[X-t \mid X>t] \\
& =\frac{\int_{t}^{+\infty}(x-t) f(x) d x}{1-F(t)}
\end{aligned}
$$

$$
\begin{aligned}
= & \frac{C(\lambda) \sum_{n=1}^{\infty} p_{n} \int_{t}^{+\infty} z g_{(n)}(z ; \alpha, n \beta, \Theta) d z}{C(\lambda)-C(\lambda G(x))}-t \\
= & \frac{C(\lambda) \sum_{n=1}^{\infty} p_{n} E\left[Z I_{(Z>t)}\right]}{C(\lambda)-C(\lambda G(x))}-t
\end{aligned}
$$

where $Z$ has a EEW distribution with parameters $\alpha, n \beta$ and $\Theta$.

\subsection{Reliability and average lifetime}

In the context of reliability, the stress-strength model describes the life of a component which has a random strength $X$ subjected to a random stress $Y$. The component fails at the instant that the stress applied to it exceeds the strength, and the component will function satisfactorily whenever $X>Y$. Hence, $R=P(X>Y)$ is a measure of component reliability. It has many applications especially in engineering concept. Here, we obtain the form for the reliability $R$ when $X$ and $Y$ are independent random variables having the same EEWPS distribution. The quantity $R$ can be expressed as

$$
\begin{aligned}
R & =\int_{0}^{\infty} f(x ; \alpha, \beta, \lambda, \boldsymbol{\Theta}) F(x ; \alpha, \beta, \lambda, \boldsymbol{\Theta}) d x \\
& =\int_{0}^{\infty} \lambda g(x) \frac{C^{\prime}(\lambda G(x)) C(\lambda G(x))}{C^{2}(\lambda)} d x \\
& =\sum_{n=1}^{\infty} p_{n} \int_{0}^{\infty} g_{(n)}(x ; \alpha, n \beta, \Theta) \frac{C(\lambda G(x))}{C(\lambda)} d x .
\end{aligned}
$$

\section{Estimation}

In this section, we first study the maximum likelihood estimations (MLE's) of the parameters. Then, we propose an Expectation-Maximization (EM) algorithm to estimate the parameters.

\subsection{The MLE's}

Let $x_{1}, \ldots, x_{n}$ be observed value from the EEWPS distribution with parameters $\xi=(\alpha, \beta, \lambda, \boldsymbol{\Theta})^{T}$. The loglikelihood function is given by

$$
\begin{aligned}
l_{n}=l_{n}(\boldsymbol{\xi} ; \boldsymbol{x})= & n[\log (\alpha)+\log (\beta)+\log (\lambda) \\
& -\log (C(\lambda))]+\sum_{i=1}^{n} \log \left[h\left(x_{i} ; \boldsymbol{\Theta}\right)\right] \\
& -\alpha \sum_{i=1}^{n} H\left(x_{i} ; \boldsymbol{\Theta}\right)+(\beta-1) \sum_{i=1}^{n} \log t_{i} \\
& +\sum_{i=1}^{n} \log \left(C^{\prime}\left(\lambda t_{i}^{\beta}\right)\right),
\end{aligned}
$$

where $x=\left(x_{1}, \ldots, x_{n}\right)$ and $t_{i}=1-e^{-\alpha H\left(x_{i} ; \boldsymbol{\Theta}\right)}$. The components of the score function $U(\xi ; x)=\left(\frac{\partial l_{n}}{\partial \alpha}, \frac{\partial l_{n}}{\partial \beta}, \frac{\partial l_{n}}{\partial \lambda}\right.$, 
$\left.\frac{\partial l_{n}}{\partial \Theta}\right)^{T}$ are

$$
\begin{aligned}
\frac{\partial l_{n}}{\partial \alpha}= & \frac{n}{\alpha}-\sum_{i=1}^{n} H\left(x_{i} ; \boldsymbol{\Theta}\right) \\
\frac{\partial l_{n}}{\partial \beta}= & \frac{n}{\beta}+\sum_{i=1}^{n} \log \left(t_{i}\right)+\sum_{i=1}^{n} \frac{\lambda t_{i}^{\beta} \log \left(t_{i}\right) C^{\prime \prime}\left(\lambda t_{i}^{\beta}\right)}{C^{\prime}\left(\lambda t_{i}^{\beta}\right)} \\
\frac{\partial l_{n}}{\partial \lambda}= & \frac{n}{\lambda}-\frac{n C^{\prime}(\lambda)}{C(\lambda)}+\sum_{i=1}^{n} \frac{t_{i}^{\beta} C^{\prime \prime}\left(\lambda t_{i}^{\beta}\right)}{C^{\prime}\left(\lambda t_{i}^{\beta}\right)} \\
\frac{\partial l_{n}}{\partial \Theta_{k}}= & \sum_{i=1}^{n} \frac{\partial h\left(x_{i} ; \boldsymbol{\Theta}\right)}{\partial \Theta_{k}} \cdot \frac{1}{h\left(x_{i} ; \boldsymbol{\Theta}\right)}-\alpha \sum_{i=1}^{n} \frac{\partial H\left(x_{i} ; \boldsymbol{\Theta}\right)}{\partial \Theta_{k}} \\
& +(\beta-1) \sum_{i=1}^{n} \frac{\partial t_{i}}{\frac{\partial \Theta_{k}}{t_{i}}}+\beta \lambda \sum_{i=1}^{n} \frac{\left[\frac{\partial t_{i}}{\partial \Theta_{k}}\right] t_{i}^{\beta-1} C^{\prime \prime}\left(\lambda t_{i}^{\beta}\right)}{C^{\prime}\left(\lambda t_{i}^{\beta}\right)}
\end{aligned}
$$

where $\Theta_{k}$ is the $k$ th element of the vector $\Theta$.

The MLE of $\xi$, say $\hat{\xi}$, is obtained by solving the nonlinear system $U(\boldsymbol{\xi} ; \boldsymbol{x})=\mathbf{0}$. We cannot get an explicit form for this nonlinear system of equations and they can be calculated by using a numerical method, like the Newton method or the bisection method. Only, for given $\Theta$, from (23) we have

$$
\alpha=\frac{n}{\sum_{i=1}^{n} H\left(x_{i} ; \boldsymbol{\Theta}\right)} .
$$

Therefore, (26) becomes

$$
\begin{aligned}
& \sum_{i=1}^{n} \frac{\partial h\left(x_{i} ; \boldsymbol{\Theta}\right)}{\partial \Theta_{k}} \cdot \frac{1}{h\left(x_{i} ; \boldsymbol{\Theta}\right)} \\
& -\frac{n}{\sum_{i=1}^{n} H\left(x_{i} ; \boldsymbol{\Theta}\right)} \sum_{i=1}^{n} \frac{\partial H\left(x_{i} ; \boldsymbol{\Theta}\right)}{\partial \Theta_{k}} \\
& +(\beta-1) \sum_{i=1}^{n} \frac{\frac{\partial t_{i}}{\partial \Theta_{k}}}{t_{i}}+\beta \lambda \sum_{i=1}^{n} \frac{\left[\frac{\partial t_{i}}{\partial \Theta_{k}}\right] t_{i}^{\beta-1} C^{\prime \prime}\left(\lambda t_{i}^{\beta}\right)}{C^{\prime}\left(\lambda t_{i}^{\beta}\right)} .
\end{aligned}
$$

Theorem 5.1. The $p d f, f(x \mid \boldsymbol{\Theta})$, of EEWPS distribution satisfies on the regularity condistions, i.e.

i. the support of $f(x \mid \boldsymbol{\Theta})$ does not depend on $\boldsymbol{\Theta}$,

ii. $f(x \mid \boldsymbol{\Theta})$ is twice continuously differentiable with respect to $\Theta$,

iii. the differentiation and integration are interchangeable in the sense that

$$
\begin{aligned}
& \frac{\partial}{\partial \boldsymbol{\Theta}} \int_{-\infty}^{\infty} f(x \mid \boldsymbol{\Theta}) d x=\int_{-\infty}^{\infty} \frac{\partial}{\partial \boldsymbol{\Theta}} f(x \mid \boldsymbol{\Theta}) d x \\
& \frac{\partial^{2}}{\partial \boldsymbol{\Theta} \partial \boldsymbol{\Theta}^{T}} \int_{-\infty}^{\infty} f(x \mid \boldsymbol{\Theta}) d x=\int_{-\infty}^{\infty} \frac{\partial^{2}}{\partial \boldsymbol{\Theta} \partial \boldsymbol{\Theta}^{T}} f(x \mid \boldsymbol{\Theta}) d x .
\end{aligned}
$$

Proof. The proof is obvious and for more details, see Casella and Berger (2001), Section 10.
The asymptotic confidence intervals of these parameters will be derived based on Fisher information matrix. It is well-known that under regularity conditions, the asymptotic distribution of $\sqrt{n}(\hat{\xi}-\xi)$ is multivariate normal with mean $\mathbf{0}$ and variance-covariance matrix $J_{n}^{-1}(\boldsymbol{\xi})$, where $J_{n}(\boldsymbol{\xi})=\lim _{n \rightarrow \infty} I_{n}(\boldsymbol{\xi})$, and $I_{n}(\boldsymbol{\xi})$ is the observed information matrix as

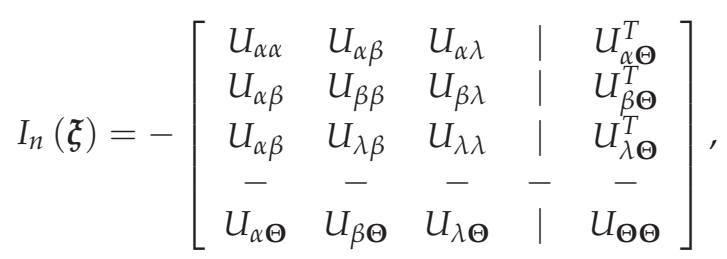

whose elements are obtained by derivative the equations (23)-(26) with respect to parameters.

\subsection{EM-algorithm}

The traditional methods to obtain the MLE's are numerical methods for solving the equations (23)-(26), and sensitive to the initial values. Therefore, we develop an EM algorithm for obtaining the MLE's of the parameters of EEWPS class of distributions. It is a very powerful tool in handling the incomplete data problem (Dempster et al., 1977). It is an iterative method, and there are two steps in each iteration: expectation step or the E-step and the Maximization step or the M-step. The EM algorithm is especially useful if the complete data set is easy to analyze.

Using (5), we define a hypothetical complete-data distribution with a joint pdf in the form

$g(x, z ; \xi)=\frac{a_{z} \lambda^{z}}{C(\lambda)} z \alpha \beta h(x ; \boldsymbol{\Theta})(1-t) t^{z \beta-1}, x>0, z \in \mathbb{N}$,

where $t=1-e^{-\alpha H(x ; \boldsymbol{\Theta})}$. The E-step of an EM cycle requires the expectation of $\left(Z \mid X ; \xi^{(r)}\right)$ where $\boldsymbol{\xi}^{(r)}=\left(\alpha^{(r)}\right.$, $\left.\beta^{(r)}, \lambda^{(r)}, \Theta^{(r)}\right)$ is the current estimate (in the $r$ th iteration) of $\xi$.

The expected value of $Z \mid X=x$ is

$$
E(Z \mid X=x)=1+\frac{\lambda t^{\alpha} C^{\prime \prime}\left(\lambda t^{\beta}\right)}{C^{\prime}\left(\lambda t^{\beta}\right)} .
$$

The M-step of EM cycle is completed by using the MLE over $\boldsymbol{\Theta}$, with the missing $z^{\prime}$ s replaced by their conditional expectations given above. Therefore, the log-likelihood for the complete-data $y=$ $\left(x_{1}, \ldots, x_{n}, z_{1}, \ldots, z_{n}\right)$ is

$$
\begin{aligned}
l^{*}(\boldsymbol{y} ; \boldsymbol{\xi}) \propto & \sum_{i=1}^{n} z_{i} \log (\lambda)+n \log (\alpha \beta)+\sum_{i=1}^{n} \log h\left(x_{i} ; \boldsymbol{\Theta}\right) \\
& -\alpha \sum_{i=1}^{n} H\left(x_{i} ; \boldsymbol{\Theta}\right)-n \log (C(\lambda)) \\
& +\sum_{i=1}^{n}\left(z_{i} \beta-1\right) \log \left(1-e^{-\alpha H\left(x_{i} ; \boldsymbol{\Theta}\right)}\right) .
\end{aligned}
$$


On differentiation of (29) with respect to parameters $\alpha$, $\beta, \lambda$ and $\Theta_{k}$, we obtain the components of the score function as

$$
\begin{aligned}
\frac{\partial l_{n}^{*}}{\partial \alpha}= & \frac{n}{\alpha}-\sum_{i=1}^{n} H\left(x_{i} ; \boldsymbol{\Theta}\right)+\sum_{i=1}^{n}\left(z_{i} \beta-1\right) \\
& \times \frac{H\left(x_{i} ; \boldsymbol{\Theta}\right) e^{-\alpha H\left(x_{i} ; \boldsymbol{\Theta}\right)}}{1-e^{-\alpha H\left(x_{i} ; \boldsymbol{\Theta}\right)}}, \\
\frac{\partial l_{n}^{*}}{\partial \beta}= & \frac{n}{\beta}+\sum_{i=1}^{n} z_{i} \log \left(1-e^{-\alpha H\left(x_{i} ; \boldsymbol{\Theta}\right)}\right), \\
\frac{\partial l_{n}^{*}}{\partial \lambda}= & \sum_{i=1}^{n} \frac{z_{i}}{\lambda}-n \frac{C^{\prime}(\lambda)}{C(\lambda)}, \\
\frac{\partial l_{n}^{*}}{\partial \Theta_{k}}= & \sum_{i=1}^{n} \frac{\partial h\left(x_{i} ; \boldsymbol{\Theta}\right)}{\partial \Theta_{k}} \cdot \frac{1}{h\left(x_{i} ; \boldsymbol{\Theta}\right)}-\alpha \sum_{i=1}^{n} \frac{\partial H\left(x_{i} ; \boldsymbol{\Theta}\right)}{\partial \Theta_{k}} \\
& +\sum_{i=1}^{n}\left(z_{i} \beta-1\right) \frac{\frac{\partial H\left(x_{i} ; \boldsymbol{\Theta}\right)}{\partial \Theta_{k}}}{1-e^{-\alpha H\left(x_{i} ; \boldsymbol{\Theta}\right)}} .
\end{aligned}
$$

Therefore, we obtain the iterative procedure of the EMalgorithm as

$$
\begin{aligned}
& \hat{\beta}^{(j+1)}=\frac{-n}{\sum_{i=1}^{n} \hat{z}_{i}^{(j)} \log \left[1-e^{-\hat{\alpha}^{(j)} H\left(x_{i} ; \hat{\mathbf{\Theta}}^{(j)}\right)}\right]}, \\
& \hat{\lambda}^{(j+1)}=\frac{C\left(\hat{\lambda}^{(j+1)}\right)}{n C^{\prime}\left(\hat{\lambda}^{(j+1)}\right)} \sum_{i=1}^{n} \hat{z}_{i}^{(j)}, \\
& \frac{n}{\hat{\alpha}^{(j+1)}}-\sum_{i=1}^{n} H\left(x_{i} ; \hat{\boldsymbol{\Theta}}^{(j)}\right)+\sum_{i=1}^{n}\left(\hat{z}_{i}^{(j)} \hat{\beta}^{(j)}-1\right) \\
& \times \frac{H\left(x_{i} ; \hat{\boldsymbol{\Theta}}^{(j)}\right) e^{-\hat{\alpha}^{(j+1)} H\left(x_{i} ; \hat{\boldsymbol{\Theta}}^{(j)}\right)}}{1-e^{-\hat{\alpha}^{(j+1)} H\left(x_{i} ; \hat{\boldsymbol{\Theta}}^{(j)}\right)}}=0, \\
& \sum_{i=1}^{n} \frac{\partial h\left(x_{i} ; \hat{\boldsymbol{\Theta}}^{(j+1)}\right)}{\partial \Theta_{k}} \cdot \frac{1}{h\left(x_{i} ; \hat{\boldsymbol{\Theta}}^{(j+1)}\right)} \\
& -\hat{\alpha}^{(j)} \sum_{i=1}^{n} \frac{\partial H\left(x_{i} ; \hat{\Theta}^{(j+1)}\right)}{\partial \Theta_{k}}
\end{aligned}
$$

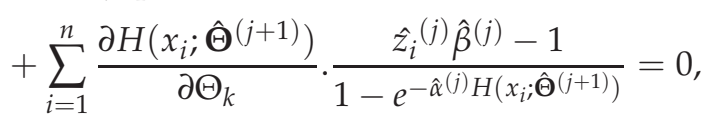

where $\hat{\lambda}^{(j+1)}, \hat{\alpha}^{(j+1)}$ and $\hat{\Theta}_{k}^{(j+1)}$ are found numerically. Here, we have

$$
\hat{z}_{i}^{(j)}=1+\frac{\lambda^{*(j)} C^{\prime \prime}\left(\lambda^{*(j)}\right)}{C^{\prime}\left(\lambda^{*(j)}\right)}, \quad i=1,2, \ldots, n,
$$

where $\lambda^{*(j)}=\hat{\lambda}^{(j)}\left[1-e^{-\hat{\alpha}^{(j)} H\left(x_{i} ; \hat{\Theta}_{k}^{(j)}\right)}\right]^{\hat{\beta}^{(j)}}$.

We can use the results of Louis (1982) to obtain the standard errors of the estimators from the EMalgorithm. Consider $\ell_{\mathcal{C}}(\boldsymbol{\Theta} ; \boldsymbol{x})=E\left(I_{\mathcal{C}}(\boldsymbol{\Theta} ; \boldsymbol{y}) \mid \boldsymbol{x}\right)$, where $I_{\mathcal{C}}(\boldsymbol{\Theta} ; \boldsymbol{y})=-\left[\frac{\partial U(\boldsymbol{y} ; \boldsymbol{\Theta})}{\partial \boldsymbol{\Theta}}\right]$ is the $(k+3) \times(k+3)$ observed information matrix. If $\ell_{m}(\boldsymbol{\Theta} ; \boldsymbol{x})=\operatorname{Var}[U(\boldsymbol{y} ; \boldsymbol{\Theta}) \mid \boldsymbol{x}]$, then, we obtain the observed information as

$$
J(\hat{\boldsymbol{\Theta}} ; \boldsymbol{x})=\ell_{c}(\hat{\boldsymbol{\Theta}} ; \boldsymbol{x})-\ell_{m}(\hat{\boldsymbol{\Theta}} ; \boldsymbol{x}) .
$$

The standard errors of the MLE's based on the EMalgorithm are the square root of the diagonal elements of the $J(\hat{\boldsymbol{\Theta}} ; \boldsymbol{x})$. The computation of these matrices are too long and tedious. Therefore, we did not present the details. Reader can see Mahmoudi and Jafari (2012) how to calculate these values.

\section{A real example}

In this section, we analyze the real data set given by Murthy et al. (2004) to demonstrate the performance of EEWPS class of distributions in practice. This data set consists of the failure times of 20 mechanical components, and is also studied by Silva et al. (2013):

$$
\begin{aligned}
& \begin{array}{l}
0.067,0.068,0.076,0.081,0.084,0.085,0.085 \\
0.086,0.089,0.098,0.098,0.114,0.114,0.115 \\
0.121,0.125,0.131,0.149,0.160,0.485
\end{array}
\end{aligned}
$$

Since the EEWPS distribution can be used for modeling of failure times, we consider this distribution for fitting these data. But, this distribution is a large class of distributions. Here, we consider five sub-models of EEWPS distribution. Some of them are suggested in literature.

(i) The exponentiated Weibull geometric (EWG) distribution, i.e. the EEWPS distribution with $H(x, \boldsymbol{\Theta})=x^{\gamma}$ and $C(\lambda)=\lambda(1-\lambda)^{-1}$.

(ii) The complementary Weibull geometric (CWG) distribution, i.e. the EEWPS distribution with $H(x, \boldsymbol{\Theta})=x^{\gamma}, C(\lambda)=\lambda(1-\lambda)^{-1}$ and $\beta=1$. This distribution is considered by Cordeiro and Silva (2014).

(iii) The generalized exponential geometric (GEG) distribution, i.e. the EEWPS distribution with $H(x, \boldsymbol{\Theta})=x$ and $C(\lambda)=\lambda(1-\lambda)^{-1}$. This distribution is considered by Mahmoudi and Tafari (2012).

(iv) The exponentiated Chen logarithmic (ECL) distribution, i.e. the EEWPS distribution with $H(x, \boldsymbol{\Theta})=\exp \left(x^{\gamma}\right)$ and $C(\lambda)=-\log (1-\lambda)$.

(v) The complementary Chen logarithmic (CCL) distribution, i.e. the EEWPS distribution with $H(x, \boldsymbol{\Theta})=\exp \left(x^{\gamma}\right), C(\lambda)=-\log (1-\lambda)$ and $\beta=1$. This distribution is considered by Cordeiro and Silva (2014).

The MLE's of the parameters for the distributions are obtained by the EM algorithm given in Section 5 Also, the standard errors of MLE's are computed and given in paracenteses. To test the goodness-offit of the distributions, we calculated the maximized 
Table 2: Parameter estimates (standard errors), K-S statistic, $p$-value, AIC, AICC, BIC, CM and AD for the data set.

\begin{tabular}{cccccc}
\hline Distribution & EWG & CWG & GEG & ECL & CCL \\
\hline$\hat{\alpha}$ & 28.665 & 25.972 & 27.752 & 17.111 & 22.019 \\
(s.e.) & $(4.617)$ & $(11.093)$ & $(6.841)$ & $(1.572)$ & $(10.177)$ \\
\hline$\hat{\gamma}$ & 0.199 & 1.642 & - & 0.136 & 1.586 \\
(s.e.) & $(0.052)$ & $(0.407)$ & - & $(0.026)$ & $(0.231)$ \\
\hline$\hat{\lambda}$ & 0.136 & 0.012 & 0.001 & 0.146 & 0.261 \\
(s.e.) & $(0.918)$ & $(1.122)$ & $(0.658)$ & $(0.032)$ & $(0.332)$ \\
\hline$\hat{\beta}$ & $5.5 e^{7}$ & - & 13.825 & $7.4 e^{7}$ & - \\
(s.e.) & $\left(1.6 e^{8}\right)$ & - & $(8.471)$ & $2.3 e^{7}$ & - \\
\hline $\log (L)$ & 37.978 & 26.422 & 32.976 & 37.794 & 25.759 \\
K-S & 0.124 & 0.264 & 0.160 & 0.121 & 0.262 \\
p-value & 0.917 & 0.122 & 0.683 & 0.931 & 0.127 \\
AIC & -67.957 & -46.845 & -59.952 & -67.588 & -45.518 \\
AICC & -65.29 & -45.345 & -58.452 & -64.922 & -44.018 \\
BIC & -63.974 & -43.858 & -56.965 & -63.606 & -42.531 \\
CM & 0.048 & 0.436 & 0.153 & 0.051 & 0.463 \\
AD & 0.402 & 2.537 & 1.136 & 0.423 & 2.663 \\
\hline
\end{tabular}

$\log$-likelihood $(\log (L))$, the Kolmogorov-Smirnov (K-S) statistic with its respective p-value, the AIC (Akaike Information Criterion), AICC (AIC with correction), BIC (Bayesian Information Criterion), CM (Cramer-von Mises statistic) and AD (Anderson-Darling statistic) for the five submodels of distribution. The $\mathrm{R}$ software (R Development Core Team, 2014) is used for the computations.

The results are given in Table 2, and from K-S, it can be concluded that all five models are appropriate for this data set. But, the EWG and ECL distributions are better than other distributions. In fact, we have a better fit when there is the parameter $\beta$ (exponentiated parameter) in model. The plots of the densities (together with the data histogram) and cdf's given in Figure 1 1 confirm this conclusion.

\section{Acknowledgments}

The authors are thankful to the referees for helpful comments and suggestions.

\section{References}

Alamatsaz, M. H. and Shams, S. (2014). Generalized linear failure rate power series distribution. Сотmunications in Statistics - Theory and Methods, In press.

Almalki, S. J. and Yuan, J. (2013). A new modified Weibull distribution. Reliability Engineering \& System Safety, 111:164-170.

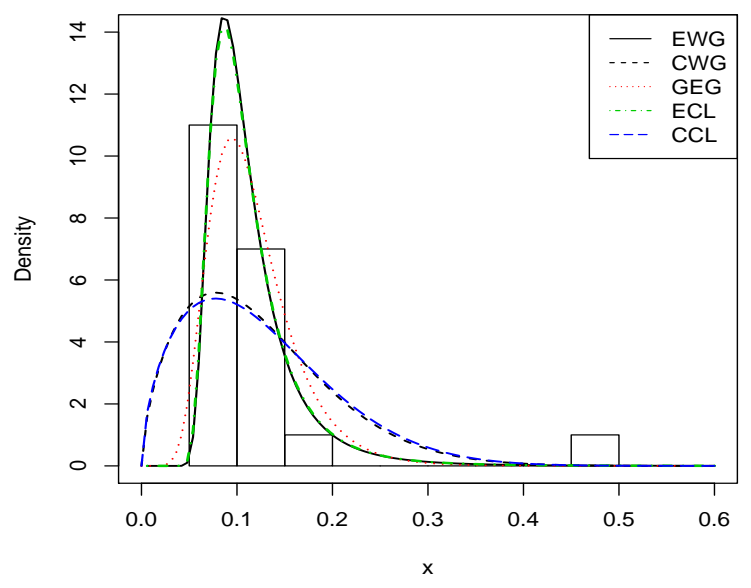

(a) Histogram

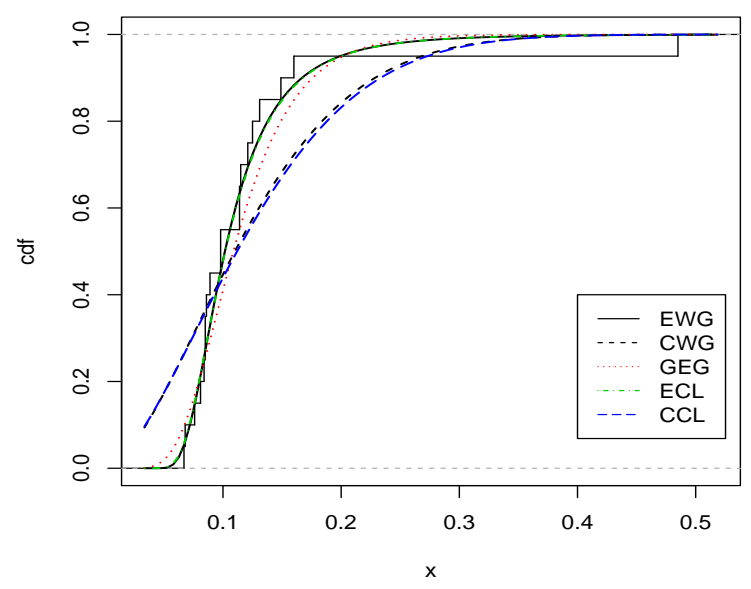

(b) Empirical distribution

Figure 1: The histogram of the data set with the estimated pdf's (a), the empirical cdf of the data set, and estimated cdf's (b) for fitted of five submodels.

Barlow, R. E. (1968). Some recent developments in reliability theory. Technical report, Unirersity of California, Berkeley.

Bidram, H., Behboodian, J., and Towhidi, M. (2013). A new generalized exponential geometric distribution. Communications in Statistics - Theory and Methods, 42(3):528-542.

Bidram, H. and Nekoukhou, V. (2013). Double bounded Kumaraswamy-power series class of distributions. Statistics and Operations Research Transactions, 37(2):211-230.

Bourguignon, M., Silva, R. B., and Cordeiro, G. M. (2014). A new class of fatigue life distributions. Journal of Statistical Computation and Simulation, 84(12): 2619-2635. 
Cancho, V. G., Louzada-Neto, F., and Barriga, G. D. C. (2011). The Poisson-exponential lifetime distribution. Computational Statistics and Data Analysis, 55(1):677686.

Carrasco, J. M., Ortega, E. M., and Cordeiro, G. M. (2008). A generalized modified Weibull distribution for lifetime modeling. Computational Statistics and Data Analysis, 53(2):450-462.

Casella, G. and Berger, R. (2001). Statistical Inference. Duxbury, Pacific Grove, California, USA.

Chahkandi, M. and Ganjali, M. (2009). On some lifetime distributions with decreasing failure rate. Computational Statistics and Data Analysis, 53(12):4433-4440.

Chen, Z. (2000). A new two-parameter lifetime distribution with bathtub shape or increasing failure rate function. Statistics \& Probability Letters, 49(2):155-161.

Cordeiro, G. M. and Lemonte, A. J. (2013). On the Marshall-Olkin extended Weibull distribution. Statistical Papers, 54(2):333-353.

Cordeiro, G. M. and Silva, R. B. (2014). The complementary extended Weibull power series class of distributions. Ciência e Natura, 36(3).

Dempster, A. P., Laird, N. M., and Rubin, D. B. (1977). Maximum likelihood from incomplete data via the EM algorithm. Journal of the Royal Statistical Society. Series B (Methodological), 39(1):1-38.

El-Gohary, A., Alshamrani, A., and Al-Otaibi, A. N. (2013). The generalized Gompertz distribution. Applied Mathematical Modelling, 37(1-2):13-24.

Flores, J., Borges, P., Cancho, V. G., and Louzada, F. (2013). The complementary exponential power series distribution. Brazilian Journal of Probability and Statistics, 27(4):565-584.

Gupta, R. D. and Kundu, D. (1999). Generalized exponential distributions. Australian $\mathcal{E}$ New Zealand Journal of Statistics, 41(2):173-188.

Jaynes, E. T. (1957). Information theory and statistical mechanics. Physical Review, 106(4):620-630.

Johnson, N. L., Kemp, A. W., and Kotz, S. (2005). Univariate Discrete Distributions. Wiley-Interscience, third edition.

Kapur, J. N. (1994). Measure of information and their Applications. John Wiley, New York.

Kundu, D. and Raqab, M. Z. (2005). Generalized Rayleigh distribution: different methods of estimations. Computational Statistics and Data Analysis, 49(1):187-200.
Lai, C., Xie, M., and Murthy, D. (2003). A modified Weibull distribution. IEEE Transactions on Reliability, 52(1):33-37.

Louis, T. A. (1982). Finding the observed information matrix when using the EM algorithm. Journal of the Royal Statistical Society. Series B (Methodological), 44(2):226-233.

Louzada, F., Marchi, V., and Carpenter, J. (2013). The complementary exponentiated exponential geometric lifetime distribution. Journal of Probability and Statistics, 2013:Article ID 502159.

Louzada, F., Marchi, V., and Roman, M. (2014). The exponentiated exponential-geometric distribution: a distribution with decreasing, increasing and unimodal failure rate. Statistics, 48(1):167-181.

Louzada, F., Roman, M., and Cancho, V. G. (2011). The complementary exponential geometric distribution: Model, properties, and a comparison with its counterpart. Computational Statistics and Data Analysis, 55(8):2516-2524.

Louzada-Neto, F., Cancho, V. G., and Barriga, G. D. C. (2011). The Poisson-exponential distribution: a Bayesian approach. Journal of Applied Statistics, 38(6):1239-1248.

Lu, W. and Shi, D. (2011). A new compounding life distribution: the Weibull-Poisson distribution. Journal of Applied Statistics, 39(1):21-38.

Mahmoudi, E. and Jafari, A. A. (2012). Generalized exponential-power series distributions. Computational Statistics and Data Analysis, 56(12):4047-4066.

Mahmoudi, E. and Jafari, A. A. (2014). The compound class of linear failure rate-power series distributions: model, properties and applications. arXiv preprint arXiv:1402.5282.

Mahmoudi, E. and Shiran, M. (2012). Exponentiated Weibull power series distributions and its applications. arXiv preprint arXiv:1212.5613.

Morais, A. L. and Barreto-Souza, W. (2011). A compound class of Weibull and power series distributions. Computational Statistics and Data Analysis, 55(3):1410-1425.

Mudholkar, G. S. and Srivastava, D. K. (1993). Exponentiated Weibull family for analyzing bathtub failurerate data. IEEE Transactions on Reliability, 42(2):299302.

Murthy, D. N. P., Xie, M., and Jiang, R. (2004). Weibull models, volume 1 . John Wiley \& Sons. 
Nadarajah, S., Cordeiro, G. M., and Ortega, E. M. M. (2015). The exponentiated G geometric family of distributions. Journal of Statistical Computation and Simulation, 85(8):1634-1650.

Nadarajah, S. and Kotz, S. (2005). On some recent modifications of Weibull distribution. IEEE Transactions on Reliability, 54(4):561-562.

Nadarajah, S., Popović, B. V., and Ristić, M. M. (2013). Compounding: an $\mathrm{R}$ package for computing continuous distributions obtained by compounding a continuous and a discrete distribution. Computational Statistics, 28(3):977-992.

Nadarajah, S., Shahsanaei, F., and Rezaei, S. (2014). A new four-parameter lifetime distribution. Journal of Statistical Computation and Simulation, 84(2):248-263.

Noack, A. (1950). A class of random variables with discrete distributions. The Annals of Mathematical Statistics, 21(1):127-132.

Pham, H. and Lai, C.-D. (2007). On recent generalizations of the Weibull distribution. IEEE Transactions on Reliability, 56(3):454-458.

R Development Core Team (2014). A Language and Environment for Statistical Computing. R Foundation for Statistical Computing, Vienna, Austria.

Roman, M., Louzada, F., Cancho, V. G., and Leite, J. G. (2012). A new long-term survival distribution for cancer data. Journal of Data Science, 10(2):241-258.

Sarhan, A. M. and Kundu, D. (2009). Generalized linear failure rate distribution. Communications in StatisticsTheory and Methods, 38(5):642-660.

Shannon, C. (1948). A mathematical theory of communication. Bell System Technical Journal, 27:379-432.

Shore, J. and Johnson, R. (1980). Axiomatic derivation of the principle of maximum entropy and the principle of minimum cross-entropy. IEEE Transactions on Information Theory, 26(1):26-37.

Silva, R. B., Bourguignon, M., Dias, C. R. B., and Cordeiro, G. M. (2013). The compound class of extended Weibull power series distributions. Computational Statistics and Data Analysis, 58:352-367.

Silva, R. B. and Cordeiro, G. M. (2013). The Burr XII power series distributions: A new compounding family. Brazilian Journal of Probability and Statistics, Accepted.

Soofi, E. S. (2000). Principal information theoretic approaches. Journal of the American Statistical Association, 95(452):1349-1353.
Surles, J. G. and Padgett, W. J. (2001). Inference for reliability and stress-strength for a scaled Burr type $X$ distribution. Lifetime Data Analysis, 7(2):187-200.

Tojeiro, C., Louzada, F., Roman, M., and Borges, P. (2014). The complementary Weibull geometric distribution. Journal of Statistical Computation and Simulation, 84(6):1345-1362.

Xie, M. and Lai, C. D. (1995). Reliability analysis using an additive weibull model with bathtub-shaped failure rate function. Reliability Engineering $\mathcal{E}$ System Safety, 52(1):87-93.

Zografos, K. and Balakrishnan, N. (2009). On families of beta- and generalized gamma-generated distributions and associated inference. Statistical Methodology, 6(4):344-362. 